\title{
Regional block: who first thought of such an approach?
}

\author{
Michiaki Yamakage $^{1}$ (i)
}

Received: 18 June 2016 / Accepted: 7 July 2016 / Published online: 12 July 2016

(C) Japanese Society of Anesthesiologists 2016

Keywords Regional block · Epidural block · Spinal block · Caudal block

The past few years have been characterized by the emergence of balanced anesthesia as a mainstream approach. Anesthetic methods that include barely achieving circulation management with nitrous oxide and volatile anesthetics and adding a small amount of fentanyl as a last resort are becoming a thing of the past. The current mainstream method involves making sufficient use of opioid analgesics such as remifentanil during surgery, with volatile anesthetics and intravenous anesthetics being regarded as sedative agents and used in the minimum amounts necessary. The advent of specific antagonists of neuromuscular blocking agents (NMBAs), such as sugammadex, has also enabled the sufficient use of rocuronium and other NMBAs during surgery. Meanwhile, although we do not know who first used this technique, a technique called regional block has long been applied. In recent years, thanks in part to advances in ultrasound sonography, many nerve blocks have come to be widely used for intraoperative and postoperative pain relief. This has also contributed to the increased popularity of balanced anesthesia and its widespread use in clinical settings. It has been a few years since the Journal of Anesthesia (JA) achieved an impact factor of over 1, and this issue also contains many outstanding manuscripts. I

Michiaki Yamakage

yamakage@sapmed.ac.jp

1 Department of Anesthesiology, Sapporo Medical University School of Medicine, South 1, West 16, Chuo-ku, Sapporo, Hokkaido 060-8543, Japan would hereby like to highlight the manuscripts relating to regional block.

Jeong et al. [1] report that the loss of resistance technique for identifying the epidural space can be practiced using a simple device created by combining a number of syringes and connectors. Given that the loss of resistance technique remains unsurpassed as the optimal approach for epidural puncture, this practice method is useful for less experienced anesthesiologists. Although their study was retrospective, Uchino et al. [2] demonstrate that the probability of migration into the intervertebral foramen clearly varies depending on the type of Tuohy needle and accompanying epidural catheter used. The authors report that the cause of these differences depends on the characteristics of the catheter tip, but, regardless of the cause, the finding that such variations arise due to differences in the catheter used is highly interesting. Meanwhile, even though ultrasound-guided techniques are being actively used in clinical settings, Yoshida et al. [3] report a case in which a catheter that was intended to be inserted into the thoracic paravertebral space migrated into the epidural space. This is certainly a case that others can learn from. Caudal block is considered a relatively safe type of epidural anesthesia. It is also performed under general anesthesia in pediatric anesthesia, and is commonly used on an outpatient basis at pain clinics. Goeller et al. [4] measured the pressure in the epidural space during bolus injection for caudal block in pediatric anesthesia. Although the pressure-which is synchronized with the heart rate-transiently increases, it then rapidly decreases to pre-injection levels. This is an interesting study that verifies the safety of caudal block from the perspective of pressure measurement. Personally, I would be interested in similar research on caudal block in adults, specifically outpatients at pain clinics. 
Let us now shift to the topic of spinal block. Based on my own experience, I am not very fond of spinal block, but it is still commonly used as an anesthetic technique, particularly in cesarean section. Hirose et al. [5] measured the concentration of total hemoglobin and the tissue oxygen index during spinal anesthesia for pregnant women undergoing cesarean section and show that both decrease in proportion to the degree of hypotension caused by bupivacaine administration. The authors attribute this finding to decreases in cerebral blood flow and oxygenation. While these results were as expected, and are events that more greatly affect the fetus, this manuscript reaffirmed the importance of managing hypotension during cesarean section. Liao et al. [6] report a case of cesarean section in a pregnant woman with uncontrolled hyperthyroidism. The anesthetic technique used was low-dose spinal and epidural anesthesia, which is a technique that I myself often use, but this pregnant woman was in a severe state (a heart rate of $117 \mathrm{bpm}$, blood pressure of 162/110 $\mathrm{mmHg}$, cardiomegaly, bronchopneumonia, and so on). In such cases, the choice of anesthetic technique is very difficult. Finally, I would like to introduce a discussion of anesthetic techniques for cesarean section. Sumikura et al. [7] provide a review of anesthetic techniques for cesarean section and conclude that, due to a combination of factors such as the paucity of experience in anesthetic techniques for cesarean section and the general increase in the ages of pregnant women, general anesthesia will not be sufficient to ensure safety in cesarean section in the future. In response, Fujita et al. [8] provide a rebuttal in the form of a Letter to the Editor. Much discussion has taken place regarding anesthetic techniques for cesarean section, including points such as the type of drug solution used in spinal anesthesia. Considering that anesthetic techniques vary greatly with facility and country, it is expected that the safety and usefulness of these techniques will continue to be actively investigated in future research.

Above, I summarized and commented on a number of manuscripts on regional block included in this issue. I selected interesting manuscripts on epidural and spinal anesthesia, which are particularly relevant given the increased use of anticoagulants during the perioperative period and the expanding indications for peripheral nerve block. The subject of regional block warrants continued attention in the future.

\section{References}

1. Jeong SM, Choi JM, Kim JH, Yoo H, Lee S, Seo H, Kim S, Kim $\mathrm{SH}$. A proposal of a simple epidural simulator for training novice anesthesiologists. J Anesth. 2016.

2. Uchino T, Miura M, Oyama Y, Matsumoto S, Shingu C, Kitano $\mathrm{T}$. Lateral deviation of four types of epidural catheters from the lumbar epidural space into the intervertebral foramen. J Anesth. 2016.

3. Yoshida T, Shimizu H, Furutani K, Baba H. Unintentional epidural placement of a thoracic paravertebral catheter inserted using an ultrasound-guided technique: a case report. J Anesth. 2016.

4. Goeller JK, Joselyn A, Martin DP, Bhalla T, Dairo O, Herz DB, Alpert SA, Tobias JD. Epidural pressure changes following caudal blockade: a prospective, observational study. J Anesth. 2016.

5. Hirose N, Kondo Y, Maeda T, Suzuki T, Yoshino A. Relationship between regional cerebral blood volume and oxygenation and blood pressure during spinal anesthesia in women undergoing cesarean section. J Anesth. 2016.

6. Liao Z, Xiong Y, Luo L. Low-dose spinal-epidural anesthesia for Cesarean section in a parturient with uncontrolled hyperthyroidism and thyrotoxic heart disease. J Anesth. 2016.

7. Sumikura H, Niwa H, Sato M, Nakamoto T, Asai T, Hagihira S. Rethinking general anesthesia for cesarean section. J Anesth. 2016;30:268-73.

8. Fujita Y, Tsuge M. Rethinking general anesthesia for cesarean section. J Anesth. 2016. 